\title{
Do deep dyslexia, dysphasia and dysgraphia share a common phonological impairment?
}

\author{
Elizabeth Jefferies*, Karen Sage, Matthew A. Lambon Ralph \\ University of Manchester, UK \\ Received 10 May 2006; received in revised form 30 November 2006; accepted 3 December 2006
}

\begin{abstract}
This study directly compared four patients who, to varying degrees, showed the characteristics of deep dyslexia, dysphasia and/or dysgraphia-i.e., they made semantic errors in oral reading, repetition and/or spelling to dictation. The "primary systems" hypothesis proposes that these different conditions result from severe impairment to a common phonological system, rather than damage to task-specific mechanisms (i.e. graphemephoneme conversion). By this view, deep dyslexic/dysphasic patients should show overlapping deficits but previous studies have not directly compared them. All four patients in the current study showed poor phonological production across different tasks, including repetition, reading aloud and spoken picture naming, in line with the primary systems hypothesis. They also showed severe deficits in tasks that required the manipulation of phonology, such as phoneme addition and deletion. Some of the characteristics of the deep syndromes - namely lexicality and imageability effects - were typically observed in all of the tasks, regardless of whether semantic errors occurred or not, suggesting that the patients' phonological deficits impacted on repetition, reading aloud and spelling to dictation in similar ways. Differences between the syndromes were accounted for by variation in other primary systems - particularly auditory processing. Deep dysphasic symptoms occurred when the impact of phonological input on spoken output was disrupted or reduced, either as a result of auditory/phonological impairment, or for patients with good phonological input analysis, when repetition was delayed. 'Deep' disorders of reading aloud, repetition and spelling can therefore be explained in terms of damage to interacting primary systems such as phonology, semantics and vision, with phonology playing a critical role.
\end{abstract}

(C) 2006 Elsevier Ltd. All rights reserved.

Keywords: Deep dyslexia; Deep dysphasia; Deep dysgraphia; Reading; Repetition; Spelling

\section{Introduction}

Deep dyslexia, deep dysphasia and deep dysgraphia (in reading aloud, repetition and spelling to dictation, respectively) have parallel features (e.g., Michel \& Andreewsky, 1983; Morton \& Patterson, 1980): (1) very severe impairment of the affected task; (2) semantic errors (e.g., cloud $\rightarrow$ "sky", which are typically taken to be the defining symptom of each condition); (3) derivational (e.g., cloud $\rightarrow$ "cloudy") and phonological/visual real-word errors (cloud $\rightarrow$ "clown"); (4) very poor performance on nonwords; (5) imageability effects-i.e., considerably better performance on concrete words (e.g., WINTER, STREAM, KITCHEN) than abstract words (e.g., REALITY, MOTIVA-

\footnotetext{
* Corresponding author at: Neuroscience and Aphasia Research Unit, School of Psychological Sciences (Zochonis Building), University of Manchester, Oxford Road, Manchester M13 9PL, UK. Tel.: +44 161275 7338; fax: +44 1612752873

E-mail address: beth.jefferies@manchester.ac.uk (E. Jefferies).
}

TION, ETIQUETTE). As these tasks involve a semi-predictable transformation from one code to another (e.g., orthography $(\mathrm{O})$ to phonology $(\mathrm{P})$ in reading aloud; auditory input $(\mathrm{A})$ to phonological output $(\mathrm{P})$ in repetition), the appearance of semantic errors is particularly striking.

Classical accounts of deep dyslexia/dysphasia propose that each of these disorders is underpinned by severe impairment to a non-lexical route devoted to reading or repetition respectively, resulting in an inability to read/repeat nonwords. This is coupled with an additional impairment affecting the lexical contribution to reading/repetition, which results in semantic errors and substantial imageability effects (Michel \& Andreewsky, 1983; Morton \& Patterson, 1980; Nolan \& Caramazza, 1982). As the non-lexical deficit in these "dual route" accounts is specific to reading (i.e., a procedure for converting graphemes to phonemes) or repetition (i.e., a mechanism for converting incoming phonology to spoken output), there should be little overlap between deep dyslexia and dysphasia. Poor grapheme-phoneme conversion in deep dyslexia should not affect repetition. Sim- 
ilarly, damage to the non-lexical repetition route would not impinge on reading aloud.

In an alternative conception, disorders of reading and repetition are caused by damage to "primary systems" - such as vision, phonology and semantics - that contribute to a multitude of language (and non-language) tasks (Farah, Stowe, \& Levinson, 1996; Patterson \& Lambon Ralph, 1999; Patterson \& Marcel, 1992; Wilshire \& Fisher, 2004). A severe impairment of phonology might underlie poor nonword reading in deep dyslexia as well as poor nonword repetition in deep dysphasia: therefore, these two conditions should have common characteristics. The "triangle" model (Plaut, McClelland, Seidenberg, \& Patterson, 1996), which proposes that reading is underpinned by interactions between distributed phonological, orthographic and semantic representations, is an instantiation of the primary systems approach. Specific disorders of reading are proposed to follow damage to particular parts of this network; for example, surface dyslexia can be modelled by damaging semantic representations whereas phonological dyslexia might follow damage to the phonological nodes.

Patients with 'phonological' dyslexia/dysphasia, like 'deep' cases, show substantial lexicality effects, but make predominantly phonological/visual as opposed to semantic errors (Beauvois \& Derouesne, 1979; Hanley \& Kay, 1997). It has been suggested that 'deep' and 'phonological' dyslexia/dysphasia lie on a continuum of severity (e.g., Crisp \& Lambon Ralph, 2006; Martin, Dell, Saffran, \& Schwartz, 1994; Wilshire \& Fisher, 2004). In line with this view, the deep dysphasic patient studied by Martin et al. (1994) showed phonological dysphasia after recovery. In addition, phonological dysphasics start to produce semantic errors when repetition is delayed (Jefferies, Crisp, \& Lambon Ralph, 2006; Martin, Saffran, \& Dell, 1996) or when several items are presented for repetition (Gold \& Kertesz, 2001; Trojano, Stanzione, \& Grossi, 1992). Crisp and Lambon Ralph (2006) found that phonological-deep dyslexic patients typically showed some of the symptoms of deep dyslexia (for example, imageability effects), whether or not they made semantic errors. In addition, Friedman (1996) noted that reading changes from a deep to a phonological pattern during recovery. This phonological-deep continuum might be underpinned by the severity of the phonological deficit alone (Patterson \& Lambon Ralph, 1999; Wilshire \& Fisher, 2004). By this view, semantic errors/imageability effects reflect the normal operation of the semantic system when non-lexical processing is stripped away (Marshall \& Newcombe, 1973; Wilshire \& Fisher, 2004). In line with this hypothesis, several studies have indicated that that patients with deep dyslexia are able to access the meanings of words they cannot read (Buchanan, McEwen, Westbury, \& Libben, 2003; Colangelo, Stephenson, Westbury, \& Buchanan, 2003; Newton \& Barry, 1997). Nevertheless, the notion that patients with deep as opposed to phonological dyslexia/dysphasia have additional semantic difficulties is compatible with the basic principles of the primary systems hypothesis, as long as the impairment affects semantic tasks in general (e.g. picture naming), rather than being specific to a lexical reading/repetition route (see Plaut \& Shallice, 1993).
What is the current evidence for a general phonological deficit in deep dyslexia? There are reports of cases who show co-occurring deficits in reading, repetition and spoken picture naming following a general impairment of phonological production (Bisiacchi, Cipolotti, \& Denes, 1989; Bub, Black, Howell, \& Kertesz, 1987; Caramazza, Miceli, \& Villa, 1986; Friedman \& Kohn, 1990; Nolan \& Caramazza, 1982)_-in severe cases, this may lead to the emergence of semantic errors in both reading and repetition (Nolan \& Caramazza, 1982). Indeed, deep dyslexia is typically associated with speech dysfluency in the context of Broca's aphasia (although a small number of cases have more fluent speech; Coltheart, 1980). Although some phonologicaldeep dyslexics can repeat items they cannot read (Nolan \& Caramazza, 1983; Patterson \& Marcel, 1977), more challenging repetition tasks, such as delayed repetition and immediate serial recall of several items, reveal greater impairment (Farah et al., 1996). When repetition is delayed, deep dyslexics show increased lexicality/imageability effects and even make semantic errors (Beland \& Mimouni, 2001; Jefferies et al., 2006). Phonological-deep dyslexics are also impaired at phonological assembly and segmentation tasks (Patterson, Suzuki, \& Wydell, 1996; Patterson \& Marcel, 1992). Their scores on such tasks correlate with both overall accuracy and imageability effects in reading (Crisp \& Lambon Ralph, 2006). Complementary research suggests that deep dyslexia results from an inability to select outputs within the phonological system (Buchanan, Hildebrandt, \& MacKinnon, 1994, 1996; Colangelo, Buchanan, \& Westbury, 2004; Katz \& Goodglass, 1990).

What about the reading abilities of deep dysphasic patients? Here the evidence is more mixed. Many deep dysphasics show surface, not deep dyslexia (Howard \& Franklin, 1988; Majerus, Lekeu, Van er Linden, \& Salmon, 2001; Valdois, Carbonnel, David, Rousset, \& Pellat, 1995). In addition, some cases have relatively good reading (Basso \& Paulin, 2003; Katz \& Goodglass, 1990). One explanation of this variation which is compatible with the primary systems approach suggests that although the same phonological representations underpin speech production in repetition and reading, repetition also draws heavily on general auditory perceptual processes: the highly systematic relationship between phonological input and output means that moderate phonological deficits may be overcome by good auditory input analysis. Consequently, some deep dysphasic patients may have impaired processing of auditoryverbal input and good phonological production on non-repetition tasks including reading (Howard \& Franklin, 1988), while others show poor phonological output in all tasks and rapid phonological decay in the context of good performance on phonological judgement tasks (Cappa, Ielasi, Tempini, \& Miozzo, 1996; Katz \& Goodglass, 1990; Martin \& Saffran, 1992; Martin, Lesch, \& Bartha, 1999).

Finally, we will consider the spelling abilities of deep dyslexics/dysphasics. Most deep dyslexics are also deep dysgraphic: i.e., they make semantic errors in spelling to dictation as well as reading (Coltheart, 1980). The primary systems hypothesis appears to provide a straightforward explanation of this finding as phonological damage should affect transcoding in both directions $(\mathrm{O} \rightarrow \mathrm{P}$ and $\mathrm{P} \rightarrow \mathrm{O})$. In contrast, if deep dyslexia is argued 
to result from damage to a specific grapheme-phoneme conversion (GPC) procedure, additional assumptions are required to account for spelling deficits (for instance, damage to a separate $\mathrm{P} \rightarrow \mathrm{O}$ process). In addition, most deep dysphasics are deep dysgraphic (Majerus et al., 2001; Valdois et al., 1995). This might reflect the fact that semantic errors can arise at any point during the translation of auditory input to a phonological code prior to the execution of spelling.

The present study focuses on the hypothesis (derived from the primary systems view) that there is a central impairment of phonology in deep dyslexia, dysphasia and dysgraphia, resulting in considerable overlap between these conditions (i.e. an impairment of repetition in deep dyslexia and difficulties with reading in deep dysphasia). Patients with these conditions have rarely (if ever) been directly compared using the same tasks and yet this comparison provides a major test of the primary systems theory. One caveat should be noted, however: variation between these patients might reflect the impact of other primary systems that contribute specifically to repetition (auditory processing) or reading (visual processing). The status of auditory processing might be especially critical given that (1) the relationship between phonological input and spoken output is more systematic than that between orthography and phonology and (2) phonological input is time-varying, unlike orthographic input, preventing patients with mild auditory processing deficits from compensating by proceeding more slowly.

We examined four patients who, to varying degrees, showed the characteristics of deep dyslexia, dysphasia and/or dysgraphia. There were three components to this investigation corresponding to the major headings in the data section below. (1) We examined the influence of lexical/semantic factors - such as lexicality, imageability and frequency - on repetition, oral reading and spelling to dictation using a single set of items. This established whether the patients showed some characteristics of the deep syndromes even for tasks in which semantic errors did not occur. (2) We assessed the status of the various "primary systems" (e.g. phonology, semantics, auditory and visual processing) which might make differential contributions to reading, repetition and spelling to dictation. This established whether differences in the deep syndromes shown by the patients could be explained in terms of the extent to which primary systems beyond phonology were impaired. (3) We employed experimental manipulations of repetition and reading to determine the ease with which semantic errors could be elicited in additional tasks. If all of the deep syndromes share a central phonological deficit and variation between them can be explained in terms of the constraints provided by additional primary systems on phonological output in different tasks (i.e., auditory and visual processing in repetition and reading respectively), manipulations that reduce the impact of these additional systems should give rise to semantic errors in additional tasks. For example, phonologically impaired deep dyslexic patients might show semantic errors in repetition under conditions in which the translation of auditory input to phonological output is difficult (e.g. following a delay). Similarly, deep dysphasic patients with phonological output deficits might be more likely to show semantic errors in reading when orthographic input is degraded. These experimental manipulations therefore provide a strong test of the primary systems hypothesis.

The primary systems view would be refuted if we failed to observe (1) a common phonological impairment in cases with deep dyslexia and deep dysphasia and (2) considerable overlap between these patients in the nature of their difficulties on reading and repetition tasks. 'Deep' symptoms such as imageability/lexicality effects and semantic errors should be present to some extent in both reading/repetition, especially following the experimental manipulations described above.

\section{Case descriptions}

The following four patients were selected for this study because they showed semantic errors in at least one task (reading, repetition or spelling to dictation). Two patients (referred to as Dyslexic-1 and Dyslexic-2) showed many features of deep dyslexia and two (Dysphasic-1 and Dysphasic-2) showed the hallmarks of deep dysphasia (all also made at least some semantic errors in spelling to dictation). In each pair, Patient 1 had more severe impairment and produced larger numbers of semantic errors in the relevant task. Their general characteristics are described below (further details of their reading, writing and repetition are provided later).

(1) Dyslexic-1 was a 66-year-old right-handed male, who left school at 15 and had previously been a sheet metal worker. He suffered a left hemisphere occipito-parietal stroke 2 years before the study. He had some loss of hearing acuity in the range $2-8 \mathrm{kHz}$, consistent with his noisy work environment. His speech was dysfluent, his repetition of single words was mildly impaired and his language comprehension was relatively intact. He scored 26/36 (50-75th percentile) on the Coloured Progressive Matrices Test of non-verbal reasoning (Raven, 1962). He made a small number of semantic errors in reading aloud and spelling to dictation although no such errors were observed in repetition.

(2) Dyslexic-2 was a 66-year-old right-handed male with a basic secondary school education who had previously worked as an undertaker. He had a left middle cerebral artery stroke 3 years before which resulted in a temporo-parietal lesion. His hearing was normal for his age, with some degree of high frequency hearing loss in both ears. His speech was fluent and free from phonemic paraphasias. His verbal comprehension was moderately impaired but his repetition was relatively preserved. He scored 30/36 (90th percentile) on the Raven's Coloured Progressive Matrices Test. He made a small number of semantic errors in reading aloud and spelling to dictation but these errors were never observed in repetition.

(3) Dysphasic-1, a 54-year-old right-handed man with a basic secondary school education, had previously worked in retail supplies and had a left temporo-parietal stroke 14 months before the study. His hearing on a pure tone audiogram 
Table 1

Repetition of words and nonwords (PALPA 9)

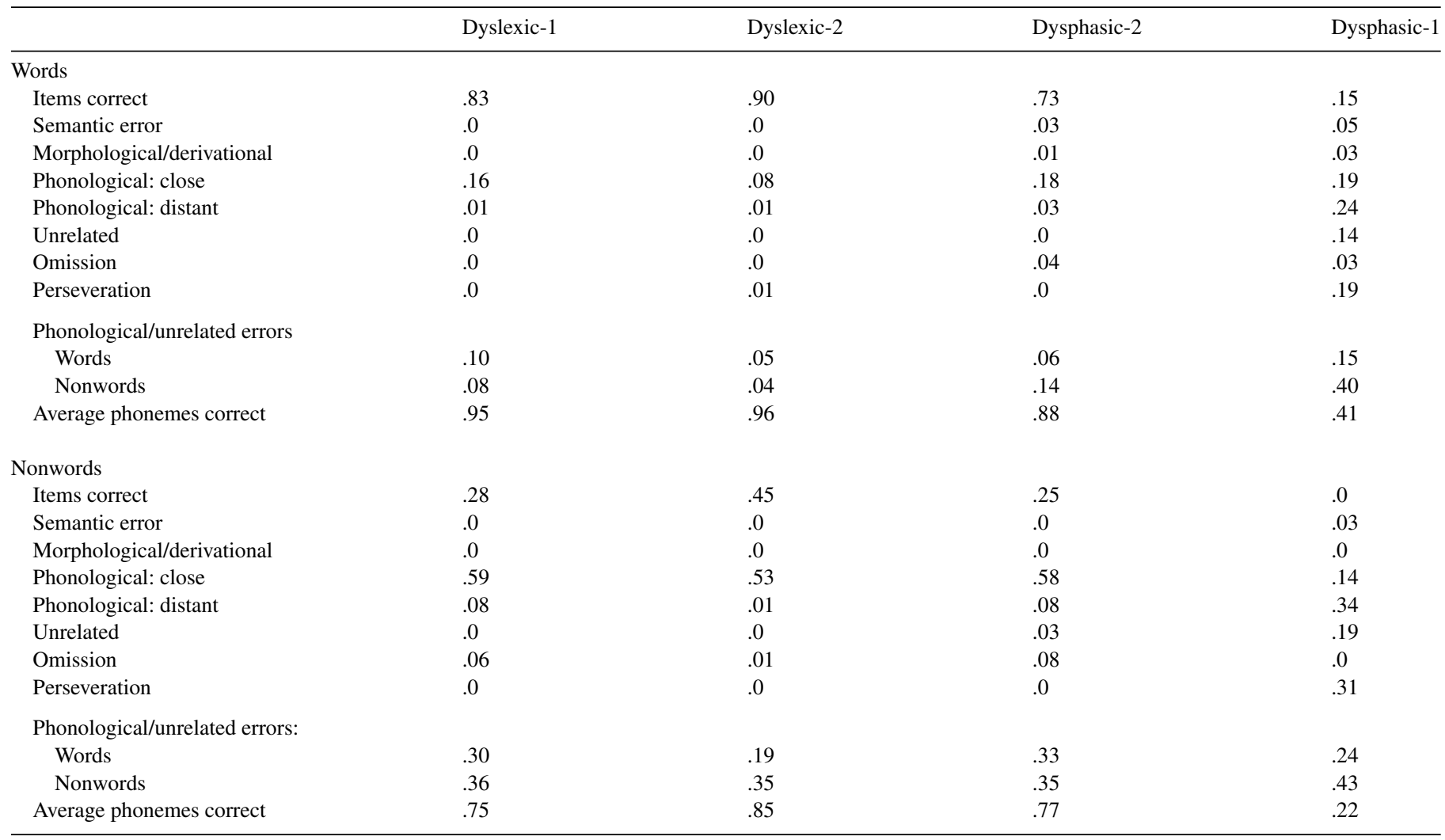

$N=80$ items. Figures show proportions of items/phonemes produced correctly and errors expressed as a proportion of trials. Data include first response only.

was normal. His spontaneous speech was relatively fluent and characterised by frequent phonological jargon. His verbal comprehension and repetition were markedly impaired. He produced semantic errors in repetition and spelling to dictation although semantic errors were never observed in reading aloud.

(4) Dysphasic-2, a 70-year-old, right-handed woman, left school at 16 and had previously worked as a cleaner. She had a large left-sided temporo-parietal stroke 5 years before the study. Her hearing was normal. Her speech was fluent and her verbal comprehension and repetition were impaired. She scored 29/36 (90th percentile) on the Raven's Coloured Progressive Matrices Test. She produced some semantically based circumlocutions in repetition, a small number of semantic errors in spelling to dictation and very occasional semantic errors in reading aloud.

The patients are always presented in the order Dyslexic-1, Dyslexic-2, Dysphasic-2, Dysphasic-1 in the data tables (i.e. a transition from deep dyslexia to deep dysphasia). Table 10 provides a summary of the neuropsychology for each patient.

\subsection{Repetition, reading and spelling to dictation}

To assess the extent of the overlap between deep dyslexia, dysphasia and dysgraphia, we examined reading, repetition and spelling to dictation in all four patients using the same items.

\subsubsection{Repetition: lexicality, imageability and frequency effects}

2.1.1.1. Method. We examined repetition using PALPA 9 (Kay, Lesser, \& Coltheart, 1992). This test contained 80 words and 80 nonwords presented in a mixed fashion. The words varied frequency and imageability orthogonally with twenty items in each set. These sets were matched for syllable length; items contained between one and four syllables. The nonwords were matched to the words for phonological complexity. The words and nonwords were spoken aloud by the experimenter. Only the first response was included in the accuracy/error analyses. ${ }^{1} \mathrm{We}$ also counted the maximum number of target phonemes produced on each trial to provide a more sensitive measure of repetition ability.

2.1.1.2. Results. Overall accuracy and errors are shown in Table 1 (see Appendix A for details of error scoring). Table 2 presents a breakdown of the results by imageability and frequency. Accuracy followed the sequence Dysphasic$1 \ll$ Dysphasic- $2<$ Dyslexic- $1<$ Dyslexic- 2 . All of the patients showed large lexicality effects (Fisher's exact test, one-tailed: $p<.0001)$. Dysphasic-1 showed a highly significant effect of imageability (Fisher's exact test, one-tailed: $p<.0001$ ), Dysphasic-2 and Dyslexic-2 showed smaller effects that reached

${ }^{1}$ Separate analyses including all responses revealed very similar findings. 
Table 2

Effect of imageability and frequency on repetition, reading and spelling to dictation (PALPA 9/40)

\begin{tabular}{|c|c|c|c|c|c|}
\hline Imageability & Frequency & Dyslexic-1 & Dyslexic-2 & Dysphasic-2 & Dysphasic-1 \\
\hline \multicolumn{6}{|c|}{ Repetition (items correct) } \\
\hline High & High & .90 & 1 & .80 & $.45(.60)$ \\
\hline High & Low & .90 & .95 & .85 & $.15(.25)$ \\
\hline Low & High & .90 & .80 & .60 & $.0(0)$ \\
\hline Low & Low & .60 & .85 & .65 & $.0(0)$ \\
\hline High & All & .90 & .98 & .83 & $.30(.43)$ \\
\hline Low & All & .75 & .83 & .63 & $.0(0)$ \\
\hline All items & & .83 & .90 & .73 & $.15(.21)$ \\
\hline \multicolumn{6}{|c|}{ Reading (items correct) } \\
\hline High & High & $.65(.90)$ & .90 & .80 & .35 \\
\hline High & Low & $.75(.90)$ & .80 & .60 & .25 \\
\hline Low & High & $.60(.75)$ & .55 & .45 & .0 \\
\hline Low & Low & $.55(.55)$ & .55 & .65 & .10 \\
\hline High & All & $.70(.90)$ & .85 & .70 & .30 \\
\hline Low & All & $.58(.65)$ & .55 & .55 & .05 \\
\hline All items & & $.64(.78)$ & .70 & .63 & .18 \\
\hline \multicolumn{6}{|c|}{ Spelling to dictation (items correct) } \\
\hline High & High & .20 & .50 & .50 & .35 \\
\hline High & Low & .30 & .35 & .35 & .25 \\
\hline Low & High & .0 & .05 & .15 & .0 \\
\hline Low & Low & .05 & .0 & .05 & .0 \\
\hline High & All & .25 & .43 & .43 & .30 \\
\hline Low & All & .03 & .03 & .10 & .0 \\
\hline All items & & .14 & .23 & .26 & .15 \\
\hline \multicolumn{6}{|c|}{ Spelling to dictation (letters correct) } \\
\hline High & High & .47 & .60 & .76 & .87 \\
\hline High & Low & .53 & .67 & .75 & .71 \\
\hline Low & High & .31 & .16 & .34 & .08 \\
\hline Low & Low & .38 & .44 & .54 & .43 \\
\hline High & All & .50 & .64 & .75 & .79 \\
\hline Low & All & .27 & .25 & .33 & .07 \\
\hline All items & & .38 & .44 & .54 & .43 \\
\hline
\end{tabular}

$N=80$ items. Figures show proportions of items/letters produced correctly on first response (self-corrections in parentheses for patients who made them frequently).

significance ( $p=.04$ and .03 , respectively) and Dyslexic-1 showed a borderline imageability effect $(p=.07)$. Dysphasic1 and Dyslexic-1 showed marginal frequency effects (Fisher's exact test, one-tailed: $p=.06$ and .07 , respectively) and Dyslexic2 and Dysphasic-2 showed little trace of a frequency effect.

The deep dysphasics, who had the poorest repetition, made small numbers of semantic and derivational errors in word repetition, largely for more imageable words (Dysphasic-1: battle $\rightarrow$ "war"; Dysphasic-2: drum $\rightarrow$ "trumpet"). They also made semantically driven responses in nonword repetition (Dysphasic-1: calt $\rightarrow$ "horse" [from colt]; Dysphasic-2: sammer $\rightarrow$ "when children finish for 6 weeks" [from summer]). Dyslexic-1, Dyslexic-2 and Dysphasic-2's repetition errors were predominantly close phonological approximations. Dysphasic-1 made a larger number of more distant phonological errors, unrelated responses and perseverations (these were typically partial reproductions of earlier responses).

2.1.1.3. Summary and discussion. All four cases were severely impaired at nonword repetition suggesting that they all had phonological deficits which might have contributed to the emergence of 'deep' errors across different tasks (e.g., reading as well as repetition). Dysphasic-1, who made the most semantic errors, showed the poorest repetition of both words/nonwords and the most marked influence of imageability. Imageability effects in repetition were also observed for the other patients, indicating that individuals with deep dyslexia show at least some of the characteristics of deep dysphasia. According to the primary systems view, the repetition of both words and nonwords is underpinned by interactivity between auditory input, semantics and phonology, so there are not two distinct routes to repetition. In line with this suggestion, we observed semantically driven responses in the nonword repetition of the two deep dysphasics (e.g., calt $\rightarrow$ "horse" [from colt]).

\subsubsection{Reading: lexicality, imageability, frequency and regularity effects}

2.1.2.1. Method. The patients' ability to read words and nonwords was assessed using PALPA 29 (letter length reading of 24 concrete words containing between three and six letters) and PALPA 36 (nonwords matched to the words for length and letter composition). The effect of frequency and imageability on word reading was assessed using the same items as for repetition above (PALPA 31). Regularity effects were examined using PALPA 35 ( $N=60$ split evenly between regular and exception words). 
Table 3

Reading aloud words and nonwords (PALPA 29/36)

\begin{tabular}{|c|c|c|c|c|}
\hline & Dyslexic-1 & Dyslexic-2 & Dysphasic-2 & Dysphasic-1 \\
\hline Items & .92 & .92 & .79 & .71 \\
\hline Mean phonemes $(S D)$ & $1(0)$ & $.96(.15)$ & $.95(.16)$ & $.83(.27)$ \\
\hline \multicolumn{5}{|l|}{ Nonwords } \\
\hline Items & .0 & .21 & .21 & .13 \\
\hline Mean phonemes $(S D)$ & $.24(.25)$ & $.76(.21)$ & $.71(.22)$ & $.55(.26)$ \\
\hline
\end{tabular}

$N=24$ items. Figures show proportions of items/phonemes produced correctly. Data include first response only.

2.1.2.2. Results. For words, accuracy followed the sequence Dyslexic-1 $>$ Dyslexic-2 $>$ Dysphasic-2 $>$ Dysphasic-1 (see Table 3). For nonwords, however, Dyslexic-1 showed a much more substantial impairment and consequently a greater lexicality effect. All four of the patients showed considerably better reading of words than nonwords (Fisher's exact test, one-tailed, $p<.0001)$. The lexicality effect for Dyslexic-1, Dyslexic-2, Dysphasic-2 and Dysphasic-1, expressed as the difference between words and nonwords, was $92 \%, 71 \%, 58 \%$ and $58 \%$, respectively (for item accuracy) and $76 \%, 20 \%, 24 \%$ and $28 \%$ (for phoneme accuracy).

Reading accuracy is shown as a function of imageability and frequency in Table 2. Dyslexic-1 often spontaneously corrected errors for high but not low imageability words: consequently, he only showed an imageability effect in reading when self-corrections were included (Fisher's exact test, one-tailed: $p=.007)$. Dyslexic-2 and Dysphasic-1 also showed significant imageability effects in reading, regardless of whether selfcorrections were included $(p<.003)$. In contrast, Dysphasic-2 did not show an influence of imageability on reading with or without self-corrections $(p>.12)$. None of the patients showed an effect of word frequency on reading accuracy $(p>.21)$. On PALPA 35, Dyslexic-1 read $73 \%$ of regular and $47 \%$ of exception words, Dyslexic-2 read 77\% of both sets, Dysphasic-2 scored $37 \%$ and $30 \%$ for regular and exception words respectively, and Dysphasic-1 scored 50\% and 23\% (excluding self-corrections). The advantage for regular words approached significance for Dysphasic-1 (Fisher's exact test: $p=.06$ ).

Table 4 shows the types of errors made by the four patients on the imageability by frequency set. The majority of errors were phonological in every case. Dyslexic-1, Dyslexic-2 and Dysphasic-2 also made small numbers of semantic errors (e.g., Dyslexic-1: hospital $\rightarrow$ "bed"; Dyslexic-2: opium $\rightarrow$ "dope"; Dysphasic-2: choir $\rightarrow$ "church") ${ }^{2}$. Compared with the other patients, Dysphasic-1 made a larger number of unrelated responses and perseverations, particularly for low imageability items (these were typically partial reproductions of earlier responses).

2.1.2.3. Summary and discussion. All of the patients showed strong effects of lexicality in reading and three of them also showed imageability effects. In a large group of patients with

\footnotetext{
${ }^{2}$ Dysphasic-2 made three semantic errors in the PALPA 35 reading test contrasting regular and irregular words.
}

phonological or deep dyslexia, Crisp and Lambon Ralph (2006) observed semantic errors in cases with more severe reading impairment. However, in our study, Dysphasic-1 did not make these errors despite having the poorest phonological production. Nevertheless, he did produce some of the characteristics of deep dyslexia, namely imageability effects. Below, we examine the hypothesis that semantic errors were masked in Dysphasic-1's reading because he still attempted to generate phonology from orthography, despite being completely unable to do so.

\subsubsection{Spelling to dictation: imageability and frequency effects}

2.1.3.1. Method. Spelling to dictation was assessed using the PALPA 31 word set described above that varied imageability and frequency. The words were spoken aloud to the patients who attempted to write them down.

2.1.3.2. Results. Accuracy was very low in this task for all four patients and followed the sequence Dysphasic $-2=$ Dyslexic$2>$ Dysphasic $-1=$ Dyslexic -1 . Table 2 shows the effects of imageability and frequency. All of the patients showed substantial imageability effects (Fisher's exact test, one-tailed, $p<.0002)$, whereas none of them showed significant effects of word frequency (Fisher's exact test, one-tailed, $p>.1$ ). As the patients' accuracy in spelling to dictation was at floor for low imageability items, we also examined the maximum number of

Table 4

Reading errors for words (PALPA 31)

\begin{tabular}{lllll}
\hline & Dyslexic-1 & Dyslexic-2 & Dysphasic-2 & Dysphasic-1 \\
\hline Items correct & .64 & .70 & .63 & .18 \\
Semantic error & .08 & .04 & .0 & .0 \\
Morphological/ & .01 & .03 & .0 & .03 \\
$\quad$ derivational & & & & \\
$\begin{array}{l}\text { Phonological: } \\
\quad \text { close }\end{array}$ & .18 & .14 & .25 & .31 \\
$\begin{array}{l}\text { Phonological: } \\
\quad \text { distant }\end{array}$ & .09 & .10 & .09 & .28 \\
Unrelated & .0 & .0 & .0 & .08 \\
$\begin{array}{l}\text { Omission } \\
\text { Perseveration }\end{array}$ & .01 & .0 & .03 & .0 \\
$\begin{array}{l}\text { Phonological/unrelated errors } \\
\quad \text { Words }\end{array}$ & .18 & .0 & .0 & .14 \\
$\quad$ Nonwords & .11 & .06 & .08 & .05 \\
\hline
\end{tabular}

$N=80$ items. Responses are expressed as a proportion of trials. Data include first response only. 
Table 5

Spelling to dictation (PALPA 31 words)

\begin{tabular}{lllll}
\hline & Dyslexic-1 & Dyslexic-2 & Dysphasic-2 & Dysphasic-1 \\
\hline Items correct & .14 & .23 & .26 & .15 \\
Semantic error & .08 & .01 & .03 & .06 \\
Morphological/ & .0 & .0 & .01 & .00 \\
$\quad$ derivational & & & & \\
Phonological/visual: & .24 & .23 & .26 & .35 \\
$\quad$ close & & & & .01 \\
Phonological/visual: & .31 & .24 & .15 & .0 \\
$\quad$ distant & .08 & .0 & .03 & .43 \\
Unrelated & .16 & .30 & .26 & .11 \\
Omission & & & & .30 \\
Phonological/unrelated errors & .14 & .06 & .04 & .34 \\
$\quad \begin{array}{l}\text { Words } \\
\quad \text { Nonwords }\end{array}$ & .41 & .31 & .03 & \\
\hline
\end{tabular}

$N=80$ items. Responses are expressed as a proportion of trials.

letters that were produced correctly on each trial. The difference between the percentage of letters correct for high and low imageability words was $72 \%, 42 \%, 39 \%$ and $23 \%$ for Dysphasic-1, Dysphasic-2, Dyslexic-2 and Dyslexic-1 respectively.

The patients' errors are shown in Table 5. All four patients made at least one semantic error; these were most common for Dysphasic-1 and Dyslexic-1. In addition, all of the patients made frequent omissions and nonword errors that were phonologically/visually related to the target word.

2.1.3.3. Summary and discussion. In line with the view that the different deep syndromes overlap, all of the patients showed some degree of deep dysgraphia. Semantic errors were most frequent for Dysphasic-1 and Dyslexic-1 who had the poorest spelling to dictation and also showed the most marked deep dysphasia/dyslexia. The imageability effect was largest for the patients who showed the most impaired oral repetition (Dysphasics-1 and 2). Like repetition, writing to dictation is heavily dependant on the ability to process spoken input, which was markedly impaired for the deep dysphasic patients (see below), especially for less imageable items that received less support from word meaning.

\subsubsection{Comparison of reading, repetition and spelling of the same items}

2.1.4.1. Method. Reading, repetition and spelling to dictation were directly compared using the PALPA 9/31 set described above. These three tasks were presented in different test sessions.

2.1.4.2. Results and discussion. The results are shown in Table 2. For the deep dyslexics, the pattern of performance was repetition $>$ reading $(\mathrm{McNemar}$, two-tailed $p<.01) \gg$ spelling to dictation $(p<.0001)$. The advantage for repetition over reading was eliminated for the deep dysphasics $(p=.19$ and .79 , respectively). Dysphasic-2 showed better reading than spelling to dictation $(p<.0001)$. Unlike the other patients, Dysphasic-1 was not significantly poorer at spelling to dictation than reading/repetition $(p=.80)$ : this might have reflected his profound phonological output deficits.

\subsection{Additional tasks to assess the status of "primary systems"}

The tasks below were administered to assess the status of each of the "primary systems" thought to contribute to repetition, reading and spelling to dictation-namely phonology, auditory processing, semantics and vision. If a common phonological impairment underlies deep dysphasia, dyslexia and dysgraphia, all four patients in this study should show substantial deficits of phonological processing. In addition, differences between reading and repetition within this group should be interpretable in terms of damage to other primary systems that contribute specifically to repetition (auditory processing) or reading (visual processing).

\subsubsection{Phonological processing}

As noted in the Introduction, moderate phonological production deficits may be overcome by good auditory input analysis in repetition due to the highly systematic relationship between phonological input and output. Therefore, deep dysphasia might be accompanied by difficulty processing spoken input. We used a variety of assessments that tapped phonological abilities; some required overt phonological production (Tests 3 and 4 below) whereas others only required verbal inputs to be processed (Tests 1 and 2). In addition, we contrasted the standard digit span task with a matching span task that did not require overt production (Test 5). Patients who have difficulty processing verbal input should have poor matching span: in contrast, patients who fail the standard digit span task because of phonological output problems should perform well.

2.2.1.1. Method. There were five tests. (1) The minimal pairs test from the PALPA required same/different judgements for pairs of monosyllabic words/nonwords that differed by a single phonetic feature (e.g., deck-neck). There were 72 trials for words and nonwords presented in separate blocks. In half the trials, the two stimuli were identical and in half they were different. The patients were allowed to respond non-verbally by pointing at a tick or cross to indicate 'same' and 'different'. (2) In a rhyme judgement task, two words were presented auditorily and the patient was required to say whether or not they rhymed (e.g., lock-dock). There were 48 trials divided equally between rhyming and non-rhyming pairs. Half of the non-rhyming words were phonologically similar. Again, the patients were allowed to respond by pointing. (3) In rhyme production, the patients were given a spoken word and were asked to produce a word that rhymed with it. There were 24 trials. (4) A phoneme segmentation task involved deletion and addition of initial phonemes of auditorily presented items (cat $\rightarrow$ at and at $\rightarrow$ cat). Equal numbers of trials involved words/nonwords at input/output, resulting in four conditions (word $\rightarrow$ word, nonword $\rightarrow$ word, word $\rightarrow$ nonword, nonword $\rightarrow$ nonword), with 24 trials in each. Tests 2-4 were taken from Patterson and Marcel (1992). (5) Finally, spoken digit span was compared with digit matching span (PALPA 13). In the standard digit span test, participants were asked to repeat strings of numbers in the correct order (e.g., " $2,6,3,1$ "). The numbers were read aloud at a rate of one 
per second. List length was gradually increased with two trials at each level. Span was taken as the longest length that could be repeated without error (at least one of the two trials correct). In the matching span test, on the other hand, two strings of digits were spoken aloud by the experimenter and a same-different judgement was required. Once again, the digits were presented at a rate of one per second with a two second pause between the two lists. On 'different' trials, the order of two adjacent numbers was reversed. Span was assessed using a stepwise procedure: longer lists were tested if the patients performed accurately and list length was decreased following an error. Span was taken as the longest length at which the majority of responses were correct.

2.2.1.2. Results. Table 6 shows the patients' scores on tests of phonological processing. On the minimal pairs test, Dyslexic1, Dyslexic-2 and Dysphasic-2 performed within or close to the normal range. In contrast, Dysphasic-1 was impaired and showed a trend towards better performance with word than nonword stimuli (Fisher's exact test, one-tailed: $p=.08$ ). The deep dysphasics showed substantial deficits in tests of rhyme judgement and production; in contrast, Dyslexic- 2 was mildly impaired and Dyslexic-1 was within the normal range. Phoneme segmentation was severely impaired in all four patients. Dyslexic-1's better performance in the word $\rightarrow$ word trials relative to the nonword $\rightarrow$ nonword trials approached significance (Fisher's exact test, two-tailed, $p=.08$ ). The other three patients were near floor in all four conditions. All four patients were impaired at spoken digit span; this deficit was most severe for Dysphasic-1. The deep dysphasics also had severe problems on the matching span task. In contrast, Dyslexic-1 performed at a more normal level on matching span.
2.2.1.3. Summary and interpretation. All four patients performed extremely poorly at the phoneme segmentation task. The fact that deep dyslexic patients show deficits on phonological tasks that do not involve reading supports the view that this condition is underpinned by a general phonological impairment (Patterson \& Marcel, 1992; Patterson et al., 1996). Nevertheless, there were differences between the patients in the degree to which phonological input processing was impaired. The deep dyslexics performed within or close to the normal range at tasks requiring analysis of spoken inputs without overt articulation (e.g., rhyme judgement, matching span). In contrast, the two deep dysphasic cases were markedly impaired. Therefore, good auditory input analysis may have been critical in preventing semantic errors in repetition for the deep dyslexics.

\subsubsection{Spoken/written picture naming}

2.2.2.1. Method. We used the Boston Naming Test $(N=60)$ to compare spoken and written picture naming.

2.2.2.2. Results. The deep dyslexics both showed a highly significant advantage for spoken over written naming (McNemar, two-tailed: $p<.0001$; see Table 7). In contrast, Dysphasic-2 showed no difference between the two conditions $(p=.45)$ and Dysphasic-1 showed a significant advantage for written over spoken naming $(p=.04)$.

The most common types of picture naming error were semantic, phonological/visual and no response errors. In spoken picture naming, Dysphasic-1 made more phonological errors than the other patients $(p<.03)$. His errors in written picture naming were more in line with those made by the other cases.

Table 6

Scores on phonological tests

\begin{tabular}{|c|c|c|c|c|c|c|}
\hline & $\operatorname{Max}$ & Dyslexic-1 & Dyslexic-2 & Dysphasic-2 & Dysphasic-1 & Normal cut-off \\
\hline PALPA minimal pairs total & 144 & 130 & 129 & 127 & $123^{*}$ & 127 \\
\hline Nonwords: same & 36 & $31^{*}$ & $33^{*}$ & 36 & $30^{*}$ & 34 \\
\hline Nonwords: different & 36 & 35 & $28^{*}$ & $29^{*}$ & $28^{*}$ & 30 \\
\hline Words: different & 36 & 29 & 33 & $28^{*}$ & 30 & 29 \\
\hline \multicolumn{7}{|l|}{$\mathrm{P} \& \mathrm{M}$ rhyme judgement } \\
\hline Non rhyming pairs & 24 & 23 & 17 & 13 & 22 & \\
\hline $\mathrm{P} \& \mathrm{M}$ rhyme production & 24 & 19 & $13^{*}$ & $7^{*}$ & $3^{*}$ & 15 \\
\hline \multicolumn{7}{|l|}{$\mathrm{P} \& \mathrm{M}$ phoneme } \\
\hline Segmentation & 96 & $37^{*}$ & $0^{*}$ & $2^{*}$ & $0^{*}$ & 76 \\
\hline Phoneme deletion & 48 & $23^{*}$ & $0^{*}$ & $2^{*}$ & $0^{*}$ & 39 \\
\hline Phoneme addition & 48 & $14^{*}$ & $0^{*}$ & $0^{*}$ & $0^{*}$ & 37 \\
\hline Spoken digit span & & $4^{*}$ & $4^{*}$ & $3^{*}$ & $1^{*}$ & 5 \\
\hline PALPA matching digit span & & 7 & 5 & 4 & 3 & \\
\hline
\end{tabular}

PALPA: Psycholinguistic Assessments of Language Processing in Aphasia (Kay et al., 1992); P\&M: Tests from Patterson and Marcel (1992).

* Indicates impaired performance. 
Table 7

Spoken and written picture naming on the Boston Naming Test

\begin{tabular}{|c|c|c|c|c|}
\hline & Dyslexic-1 & Dyslexic- 2 & Dysphasic-2 & Dysphasic-1 \\
\hline \multicolumn{5}{|l|}{ Spoken naming } \\
\hline Items correct (self-corrections) & $.38(.08)$ & $.50(.08)$ & $.35(.13)$ & $.13(.03)$ \\
\hline Semantic error & .32 & .22 & .28 & .17 \\
\hline Morphological/derivational & .02 & .03 & .0 & .02 \\
\hline Phonological: close & .08 & .10 & .18 & .27 \\
\hline Phonological: distant & .07 & .07 & .08 & .30 \\
\hline Unrelated & .0 & .0 & .0 & .07 \\
\hline Omission & .13 & .03 & .10 & .03 \\
\hline Perseveration & .0 & .05 & .0 & .02 \\
\hline \multicolumn{5}{|l|}{ Phonological/unrelated errors } \\
\hline Words & .05 & .05 & .07 & .08 \\
\hline Nonwords & .07 & .10 & .17 & .43 \\
\hline \multicolumn{5}{|l|}{ Written naming } \\
\hline Items correct & .10 & .18 & .28 & .25 \\
\hline Semantic error & .12 & .12 & .08 & .13 \\
\hline Morphological/derivational & .0 & .0 & .03 & .02 \\
\hline Visual: close & .20 & .28 & .18 & .32 \\
\hline Visual: distant & .43 & .23 & .18 & .12 \\
\hline Unrelated & .1 & .03 & .0 & .0 \\
\hline Omission & .03 & .15 & .23 & .17 \\
\hline Perseveration & .02 & .0 & .0 & .0 \\
\hline \multicolumn{5}{|l|}{ Visual/unrelated errors } \\
\hline Words & .12 & .07 & .03 & .0 \\
\hline Nonwords & .62 & .45 & .23 & .42 \\
\hline
\end{tabular}

$N=60$ items. Figures show proportions of trials. Data include first response only (because of the high rate of self-corrections in spoken naming, these responses are also indicated).

\subsubsection{Summary and discussion. Although all four patients} were markedly impaired at both spoken and written naming, the relative severity of these two deficits varied. The deep dyslexics had greater difficulty in written than spoken picture naming whereas the deep dysphasics did not show this difference. Dysphasic-1 made more phonological errors than the other patients in spoken picture naming and was the only patient to show more accurate written than spoken naming, suggesting that he had a more severe impairment of phonological output.

\subsubsection{Visual-spatial processing}

The patients' CT scans revealed that Dyslexic-1 had occipito-parietal damage, whereas the other patients had temporal-parietal damage: consequently, Dyslexic-1 might have had more severe visual-spatial processing deficits which could have contributed to his reading difficulties. We therefore assessed visual-spatial processing using the three tests below.

2.2.3.1. Method. (1) The VOSP battery (Visual Object and Space Perception; Warrington \& James, 1991) was used to assess a range of visual and spatial skills. (2) The patients attempted to copy the Rey complex figure without a delay (taken from Lezak, 1976). This test tapped planning abilities and spatial attention. (3) We used the Map Search task from the Test of Everyday Attention. This involved searching for a particular symbol (e.g., a knife and fork, signifying a restaurant) on a map: subjects marked each instance of the symbol with a pen and were given a time limit of 2 min. We were not able to test Dysphasic-1 on Tests 2 and 3.
2.2.3.2. Results. (1) On the VOSP, all four cases were in the normal range on the majority of subtests (screening, incomplete letters, object decision, progressive silhouettes, dot counting and number location). Dyslexic-1 was below the normal range for position discrimination $(16 / 20$; cut-off $=18)$ and Dysphasic-1 was below the normal range for cube analysis $(4 / 10$; cut-off $=6)$, although this possibly reflected cognitive or comprehension difficulties rather than a visuospatial deficit per se. (2) Dyslexic-1 and Dysphasic-2 showed some weakness on the Rey figure copy (25/36 and 22/36, respectively). Dyslexic-1's copy was extremely slow and done in a piecemeal fashion, whereas Dysphasic-2's copy was rapid and slapdash. Dyslexic-2 performed relatively well on this task (33/36). (3) Dyslexic-1 was profoundly impaired at the map search task (score $=9,<1$ st percentile). Dyslexic-2 and Dysphasic-2 also showed somewhat weak performance: they scored 40 (12th percentile) and 35 (7th percentile), respectively.

2.2.3.3. Summary. Dyslexic-1 was the only case who was impaired on a simple spatial processing task (position discrimination). He was also more impaired than the other patients on the demanding map search task.

\subsubsection{Letter knowledge tasks}

2.2.4.1. Method. There were four tasks. The first two involved phonological input and orthographic output: (1) writing the 26 letters in a random order in response to their spoken names; (2) selecting the 26 written letters in response to their spoken names 
Table 8

Letter knowledge tests

\begin{tabular}{|c|c|c|c|c|c|}
\hline & Max & Dyslexic-1 & Dyslexic-2 & Dysphasic-2 & Dysphasic-1 \\
\hline Spoken-written letter match & 26 & 19 & 23 & 18 & 0 \\
\hline Writing letters to dictation & 26 & 15 & 19 & 20 & 7 \\
\hline Cross-case strings match (PALPA 23) & 60 & 54 & 57 & 57 & NT \\
\hline
\end{tabular}

NT: not tested.

(PALPA 23; there were four choices on each trial). The second two tasks did not involve phonological input: (3) the highest score on letter naming ( $\rightarrow$ "tee") or sounding $(\mathrm{T} \rightarrow$ " $/ \mathrm{t} / \mathrm{l}$ ") was taken as an indication of the patients' ability to recognise orthographic forms $(N=26)$; (4) the patients were asked to make same-different judgements in a cross-case matching task for strings of letters that were either words (dream-DREAD) or nonwords (ckitr-CRTAE; PALPA 21). In this test, there were 60 trials, half of which were different.

2.2.4.2. Results. Dyslexic-2 and Dysphasic-2 showed mild deficits on all four tasks (see Table 8). Dysphasic-1 was more severely impaired than the other three cases at the tasks that involved processing auditory verbal input, namely spoken-written letter matching and writing letters to dictation. Dysphasic-1's letter sounding abilities were only mildly impaired. In contrast, Dyslexic-1 was more severely impaired at the two tasks that involved visual/orthographic input (cross-case matching, letter naming/sounding).

\subsubsection{Semantic memory}

Deficits of semantic memory might affect the manifestation of deep syndromes in reading, repetition and spelling (see Section 1). Some accounts suggest that differences between deep dyslexic patients are explicable in terms of the nature or severity of the semantic impairment (Dickerson \& Johnson, 2004; Shallice \& Warrington, 1980). Therefore, we examined the performance of our patients on a variety of comprehension tasks. The tasks compared comprehension of (a) verbal and non-verbal stimuli, (b) concrete and abstract words and (c) spoken and written words.

2.2.5.1. Method. The patients were tested on (1) two picture association tasks: the Pyramids and Palm Trees test (Howard \& Patterson, 1992) and the Camel and Cactus Test (Bozeat, Lambon Ralph, Patterson, Garrard, \& Hodges, 2000). These involved choosing the picture that was most associated with a target. In the CCT, there were 64 trials, each with four choices (e.g., does 'camel' go with 'cactus', 'tree', 'sunflower' or 'rose'?). In the PPT, there were two choices and 52 trials. We also examined judgements of semantic association for word stimuli using the CCT items. In this test, written words were presented instead of pictures and these were read aloud by the experimenter. (2) Two synonym judgement tests contrasted comprehension of concrete/imageable and abstract items (Warrington, McKenna, \& Orpwood, 1998). In the graded synonym test of Warrington et al., there were 25 concrete and abstract trials presented in sepa- rate blocks, with two choices per trial (i.e., does 'wager' go with 'bet' or 'cost'?). The distracter words were semantically related to the correct response. In the synonym test from the PALPA battery, there were 60 trials divided equally between high and low imageability trials presented in a mixed fashion. Patients were asked to judge if pairs of words had broadly similar meanings or not (i.e., 'story' with 'tale'; yes or no?). For both tests, we compared the comprehension of spoken and written words in two separate presentations. (3) Auditory word-picture matching was assessed using the test from the ADA battery (Franklin, Turner, \& Ellis, 1992). There were 66 trials in which the patients had to select a target picture matching an auditorily presented word from an array containing five distracters (one semantically related to the target, one phonologically related, one both semantically and phonologically related and two unrelated). (4) The patients were asked to match environmental sounds (e.g., a telephone ringing) to pictures/written words (Bozeat et al., 2000). There were 48 trials in both versions of the test, each with ten semantically related choices.

2.2.5.2. Results. Table 9 shows the patients' scores. All four patients performed within or close to the normal range on picture and word tests of semantic association. However, three of the patients (Dyslexic-2, Dysphasics-1 and 2) showed some weakness on tests of synonym judgement using both auditory and written presentation (Dyslexic-1 was within or close to the normal range). Two of these patients, Dyslexic-2 and Dysphasic-1, performed significantly more poorly with spoken than written words (Fisher's exact test, two-tailed $p<.0002$ ) and all three made more errors with abstract than concrete/imageable words (Fisher's exact test, one-tailed, $p<.05$, combining scores across two tests). This imageability effect came largely from the auditory presentation condition. Dysphasic-1 was also impaired at word-picture matching; the other three patients were within or close to the normal range. Dyslexic-1, Dyslexic-2 and Dysphasic-2 were impaired at matching environmental sounds to pictures. Dyslexic-1 and Dyslexic-2 also showed substantial weakness on sound-word matching.

\subsubsection{Summary and interpretation}

The patients did not have a marked semantic impairment in general because they performed normally or close to the normal range on verbal and non-verbal tests of semantic association (PPT and CCT). However, Dysphasic-1, Dyslexic-2 and Dysphasic-2 were impaired at synonym judgement, especially for spoken, abstract words and Dysphasic-1's profound difficulty understanding spoken words extended to word-picture 
Table 9

Scores on semantic tests

\begin{tabular}{|c|c|c|c|c|c|c|}
\hline & Max & Dyslexic-1 & Dyslexic-2 & Dysphasic-2 & Dysphasic-1 & Normal cut-off \\
\hline PPT picture & 52 & $48^{*}$ & 49 & $48^{*}$ & $48^{*}$ & 49 \\
\hline CCT picture & 64 & 51 & 51 & 52 & 56 & 51 \\
\hline CCT word & 64 & $53^{*}$ & $54^{*}$ & 60 & 60 & 56 \\
\hline Warrington auditory synonym & 50 & 36 & $21^{*}$ & 31 & $22^{*}$ & 29 \\
\hline Concrete & 25 & 17 & 16 & 15 & 15 & 14 \\
\hline Abstract & 25 & 19 & $5^{*}$ & 16 & $7^{*}$ & 14 \\
\hline Warrington written synonym & 50 & 42 & $36^{*}$ & $36^{*}$ & $34^{*}$ & $42^{\mathrm{a}}$ \\
\hline Concrete & 25 & 21 & $19^{*}$ & $20^{*}$ & $16^{*}$ & $21^{\mathrm{a}}$ \\
\hline Abstract & 25 & 21 & $17^{*}$ & $16^{*}$ & $18^{*}$ & $20^{\mathrm{a}}$ \\
\hline PALPA auditory synonym & 60 & $54^{*}$ & $41^{*}$ & $48^{*}$ & $41^{*}$ & 56 \\
\hline High imageability & 30 & 28 & $24^{*}$ & $27^{*}$ & $25^{*}$ & 28 \\
\hline Low imageability & 30 & $26^{*}$ & $17^{*}$ & $21^{*}$ & $21^{*}$ & 27 \\
\hline PALPA written synonym & 60 & $55^{*}$ & $55^{*}$ & $54^{*}$ & $54^{*}$ & 56 \\
\hline High imageability & 30 & $29^{*}$ & $29^{*}$ & $28^{*}$ & $29^{*}$ & 30 \\
\hline Low imageability & 30 & 26 & 26 & 26 & $25^{*}$ & 26 \\
\hline Combined auditory synonym & 110 & 90 & $62^{*}$ & $79^{*}$ & $63^{*}$ & 85 \\
\hline High imageability/concrete & 55 & 45 & $40^{*}$ & 42 & $40^{*}$ & 42 \\
\hline Low imageability/abstract & 55 & 45 & $22^{*}$ & $37^{*}$ & $28^{*}$ & 41 \\
\hline Combined written synonym & 110 & 97 & $91^{*}$ & $90^{*}$ & $88^{*}$ & 98 \\
\hline High imageability/concrete & 55 & 50 & $48^{*}$ & $48^{*}$ & $45^{*}$ & 51 \\
\hline Low imageability/abstract & 55 & 47 & $43^{*}$ & $42^{*}$ & $43^{*}$ & 46 \\
\hline ADA spoken word-picture match & 66 & $60^{*}$ & $60^{*}$ & 64 & $47^{*}$ & 63 \\
\hline Environmental sound-picture match & 48 & $31^{*}$ & $29^{*}$ & $27^{*}$ & 43 & 38 \\
\hline Environmental sound-word match & 48 & $31^{*}$ & $28^{*}$ & $36^{*}$ & $37^{*}$ & 38 \\
\hline
\end{tabular}

PPT: Pyramids and Palm Trees Test (Howard \& Patterson, 1992); CCT: Camel and Cactus Test (Bozeat et al., 2000); Warrington Synonym Test: (Warrington et al., 1998); PALPA: Psycholinguistic Assessments of Language Processing in Aphasia (Kay et al., 1992); Environmental sounds test (Bozeat et al., 2000); ADA: (Franklin et al., 1992).

* Patient scores that are impaired.

${ }^{\text {a }}$ Norms from Sage and Ellis (2004).

matching. These difficulties may have reflected the patients' phonological problems because synonym judgement makes significant demands on phonological maintenance, particularly for abstract words (Binder, Westbury, McKiernan, Possing, \& Medler, 2005; Westbury \& Buchanan, 2006). Our results do not provide any positive evidence to support the view that deep dys- phasia and dyslexia necessarily involve multiple impairments of phonology and semantics because the symptoms of deep dyslexia/dysphasia did not co-occur with greater impairment on semantic tasks. Dyslexic-1 showed the best verbal comprehension and yet showed the symptoms of deep dyslexia most strongly. In addition, spoken comprehension on synonym

Table 10

Summary of patients' deficits

\begin{tabular}{|c|c|c|c|c|}
\hline & Dyslexic-1 & Dyslexic-2 & Dysphasic-2 & Dysphasic-1 \\
\hline Repetition accuracy & $x$ & $x$ & $x$ & $x \times$ \\
\hline Lexicality effect in repetition & $\mathrm{P}$ & $\mathrm{P}$ & $\mathrm{P}$ & $\mathrm{P}$ \\
\hline Imageability effect in repetition & $\mathrm{P} ?$ & $\mathrm{P}$ & $\mathrm{P}$ & PP \\
\hline Semantic errors in repetition & A & A & $\mathrm{P}$ & $\mathrm{P}$ \\
\hline Reading accuracy & $x$ & $x$ & $x$ & $x \times$ \\
\hline Lexicality effect in reading & PP & $\mathrm{P}$ & $\mathrm{P}$ & $\mathrm{P}$ \\
\hline Imageability effect in reading & $\mathrm{P}$ & $\mathrm{P}$ & A & $\mathrm{P}$ \\
\hline Semantic errors in reading & $\mathrm{P}$ & $\mathrm{P}$ & A & A \\
\hline Spelling to dictation accuracy & $x \times$ & $\times$ & $x$ & $x \times$ \\
\hline Imageability effect in spelling & $\mathrm{P}$ & $\mathrm{P}$ & $\mathrm{P}$ & PP \\
\hline Semantic errors in spelling & PP & $\mathrm{P}$ & $P$ & PP \\
\hline Phonology: input & $\sqrt{ } ?$ & $\times ?$ & $x$ & $x \times$ \\
\hline Phonology: output & $x$ & $x$ & $\times$ & $x \times$ \\
\hline Visual-spatial processing & $\times$ & $\sqrt{ }$ & $\sqrt{ }$ & $\sqrt{ } ?$ \\
\hline Single letter tasks: P-O & $\times$ & $\times$ & $\times$ & $x \times$ \\
\hline Single letter tasks: O-P & $x \times$ & $x$ & $x$ & $x$ \\
\hline Non-verbal comprehension & $\sqrt{ } ?$ & $\sqrt{ } ?$ & $\sqrt{ } ?$ & $\sqrt{ }$ \\
\hline Verbal comprehension & $\sqrt{ } ?$ & $\times$ & $\times$ & $\times$ \\
\hline
\end{tabular}

$\times \times$ : very impaired; $\times$ : impaired; $\sqrt{ }$ ?: largely intact; $\sqrt{ }$ : intact; PP: strongly present; P: present; A: absent. 
judgement tasks was poorer for Dyslexic-2 than Dysphasic2; nevertheless Dysphasic-2 showed deep dysphasia whereas Dyslexic-2 had relatively good repetition.

\subsubsection{Summary of deficits}

Table 10 summarises the patients' performance. All four patients had substantial phonological production deficits: they performed poorly across different tasks requiring spoken responses, including repetition, reading aloud and spoken picture naming. They were also severely impaired at a phoneme segmentation task. These findings are consistent with the primary systems view that a common phonological deficit underlies the different deep syndromes. Dysphasic-1's phonological output problem was particularly severe: his accuracy was lowest on all the tasks requiring spoken output and he was the only patient to show better written than spoken picture naming. The patients showed some of the hallmarks of the deep syndromes (i.e. lexicality and imageability effects) even in tasks that were free from semantic errors, supporting the view that there is considerable overlap between these conditions. However, there were differences between the cases that appeared to be explicable in terms of the contribution of additional primary systems. Deep dysphasia (i.e., the production of semantic errors in repetition) was most marked in the patients who showed clear difficulty with the analysis of spoken input. The better auditory input analysis of the deep dyslexics may have prevented semantic errors in repetition, despite these patients' clear phonological deficits, because there is a highly systematic relationship between auditory input and phonology. This might have allowed the spoken form of a word to specify the appropriate phonological output during repetition, overcoming these patients' difficulties in phonological processing. Consequently, manipulations of repetition that weaken the ability of auditory input to influence phonological output (such as the inclusion of a delay between input and output) might elicit deep dysphasic symptoms in the deep dyslexics.

\subsection{Experimental manipulations designed to elicit semantic errors in additional tasks}

\subsubsection{Manipulations of repetition}

Semantic errors might occur in repetition when phonological input cannot strongly constrain phonological output due to impaired auditory-verbal processing (e.g., Howard \& Franklin, 1988) and/or rapid phonological decay (Martin \& Saffran, 1992; Martin et al., 1996). Consequently, if the standard repetition task is manipulated to reduce the influence of spoken input on phonological output, patients like Dyslexics-1 and 2, with impaired phonological production in the context of good analysis of spoken input, might start to show the hallmarks of deep dysphasia (e.g., imageability effects and semantic errors; see Beland \& Mimouni, 2001; Farah et al., 1996; Jefferies et al., 2006). We examined repetition under three conditions: immediate single word, delayed single word and word pair repetition. In delayed and paired repetition, the input was less able to constrain output because non-target phonological processing occurred prior to the response.
Table 11

Manipulations of repetition

\begin{tabular}{|c|c|c|c|}
\hline & $\begin{array}{l}\text { Immediate } \\
\text { single word }\end{array}$ & $\begin{array}{l}\text { Delayed } \\
\text { single word }\end{array}$ & $\begin{array}{l}\text { Immediate } \\
\text { word pair }\end{array}$ \\
\hline \multicolumn{4}{|l|}{ Dyslexic-1 } \\
\hline High imageability & 1.0 & .81 & .66 \\
\hline Low imageability & .91 & .50 & .41 \\
\hline Imageability difference & .09 & .31 & .25 \\
\hline Semantic errors & .0 & .06 & .0 \\
\hline \multicolumn{4}{|l|}{ Dyslexic-2 } \\
\hline High imageability & .88 & .63 & .81 \\
\hline Low imageability & 1.0 & .16 & .59 \\
\hline Imageability difference & -.13 & .47 & .22 \\
\hline Semantic errors & .0 & .0 & .0 \\
\hline \multicolumn{4}{|l|}{ Dysphasic-2 } \\
\hline High imageability & .94 & .84 & .56 \\
\hline Low imageability & .75 & .25 & .34 \\
\hline Imageability difference & .19 & .59 & .22 \\
\hline Semantic errors & .03 & .16 & .13 \\
\hline \multicolumn{4}{|l|}{ Dysphasic-1 } \\
\hline High imageability & .38 & .44 & .31 \\
\hline Low imageability & .16 & .0 & .0 \\
\hline Imageability difference & .22 & .44 & .31 \\
\hline Semantic errors & .22 & .22 & .34 \\
\hline
\end{tabular}

Data expressed as a proportion of trials $(N=64$ in total), including selfcorrections.

2.3.1.1. Method. There were 64 words that crossed imageability and frequency, with equal numbers of items in each set. Repetition was examined in three conditions: (1) immediate single word repetition; (2) repetition after a delay of approximately $10 \mathrm{~s}$ filled by the subject and experimenter counting together up to 15 ; (3) immediate repetition of a pair of words drawn from the same frequency by imageability category. These three conditions were tested in different sessions.

2.3.1.2. Results. Table 11 shows the patients' accuracy in each condition and the total number of semantic errors as a proportion of the number of trials. Dyslexics-1 and 2 and Dysphasic- 2 all showed less accurate repetition in the delayed/paired conditions than in the immediate condition (McNemar test, exact one-tailed $p<.001)$. In contrast, Dysphasic- 1 showed no difference between immediate and delayed repetition of a single item $(p=.30)$ and the difference between single versus paired repetition was smaller than that seen in the other cases $(p=.03)$. This presumably reflected a floor effect given Dysphasic-1's very poor repetition.

The deep dyslexic patients showed significant imageability effects in delayed repetition (Fisher's exact test: $p=.008$ and .0001) and word-pair repetition ( $p=.04$ and .05$)$ but not immediate repetition ( $p=.12$ and .06). The deep dysphasics had poorer immediate repetition of single words and showed imageability effects in every condition (immediate: $p=.04$; delayed: $p<.0001$; paired: $p=.0004$ for Dysphasic-1 and $p=.07$ for Dysphasic-2). The deep dysphasics produced semantic errors in every condition. Dyslexic-1 made occasional semantic errors in delayed repetition (none were observed in standard repetition tasks). Dyslexic-2 did not show semantic errors. 
alcohol

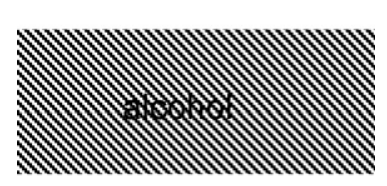

AlCoHoL

Fig. 1. Manipulations of reading.

2.3.1.3. Summary. The deep dyslexics made few errors in immediate single word repetition but showed much poorer delayed/paired repetition when phonological input was unable to strongly constrain spoken output (cf. Farah et al., 1996; Jefferies et al., 2006). In delayed/paired repetition, the deep dyslexics showed more substantial imageability effects and Dyslexic-1 also made occasional semantic errors; consequently, a reduction in support from auditory input produced some of the hallmarks of deep dysphasia.

\subsubsection{Manipulations of reading}

If deep dyslexia has its roots in severe deficits of phonological production, we need to explain why semantic errors were not evident in Dysphasic-1's reading. One possibility is that semantic errors were blocked by Dysphasic-1's (unsuccessful) attempts to activate phonology from orthography. Similar arguments have been put forward previously by Hillis and Caramazza (1991) and Marshall and Newcombe (1973). We predicted that manipulations that reduced the activation of phonology from orthography would elicit deep dyslexic symptoms for Dysphasic-1. We examined the influence of two manipulations: masked presentation and alternating case (see Fig. 1). The first of these manipulations masked both global word shape and the features of individual letters, whereas the second only disrupted global word shape.

2.3.2.1. Method. In a pilot study, we compared normal presentation with masked reading $(N=15)$ and alternating case $(N=25)$ for each patient. The masked presentation involved presenting words through a panel of diagonal black lines (see Fig. 1). The alternating case manipulation required the patients to read words that were composed of letters that switched between upper and lower case. We took the manipulation that produced the largest effect for each patient in this pilot study (alternating case for Dyslexic-1, Dyslexic-2 and Dysphasic-2; masked presentation for Dysphasic-1) and examined its impact on words varying in imageability/frequency using the set of items described above from PALPA $31(N=80)$.

2.3.2.2. Results. In the pilot study, alternating case had a substantial impact on Dyslexic-1's reading (accuracy: $64 \%$ vs. $28 \%$ correct, McNemar, exact one-tailed $p=.006$; semantic errors as a proportion of trials, $0 \%$ vs. $16 \%$ ), but no significant effect for Dyslexic-2 (64\% vs. $52 \%$ correct; $0 \%$ vs. $12 \%$ semantic errors), Dysphasic-2 (52\% vs. $56 \%$ correct; $12 \%$ vs. $12 \%$ semantic errors) or Dysphasic-1 (4\% vs. $4 \%$ correct; $0 \%$ vs. $8 \%$ semantic errors). Masked presentation had comparatively little effect for Dyslexic-1 (53\% vs. $33 \%$ correct, McNemar n.s.; $13 \%$ vs. $7 \%$ semantic errors), Dyslexic-2 (60\% vs. $67 \%$ correct; $0 \%$ vs. $7 \%$ semantic errors) or Dysphasic-2 (47\% vs. $67 \%$ correct; $0 \%$ vs. $7 \%$ semantic errors). However, the mask dramatically increased the rate of semantically driven responses for Dysphasic-1 (0\% vs. $93 \%$ trials; accuracy was $0 \%$ in both conditions). Many of these responses were semantic descriptions (QUALITY $\rightarrow$ "it is good stuff"). Self-corrections and semantic responses that occurred in conjunction with other errors were included in these counts.

Table 12 shows the four patients' performance on the PALPA 31 items. Reading accuracy was significantly lower in the alternating case condition for Dyslexic-1 (McNemar, exact onetailed $p=.013$ ) but not for Dyslexic-2 and Dysphasic-2. For Dysphasic-1, masked presentation increased the rate of semantic errors/descriptions from $0 \%$ to $38 \%$ of trials (exact two-tailed $p<.0001$ ). Surprisingly, Dysphasic-1's reading accuracy was also higher for masked than normal presentation (McNemar, exact two-tailed $p=.027)$. This effect was entirely carried by the high frequency items (McNemar, exact two-sided $p=.035$ ). The reading manipulations did not substantially alter the size of the imageability effect for any of the patients.

Table 12

Manipulations of reading

\begin{tabular}{|c|c|c|c|c|c|c|c|c|}
\hline & \multicolumn{2}{|c|}{ Dyslexic-1 } & \multicolumn{2}{|c|}{ Dyslexic-2 } & \multicolumn{2}{|c|}{ Dyslexic-2 } & \multicolumn{2}{|c|}{ Dysphasic-1 } \\
\hline & Normal & Alt case & Normal & Alt case & Normal & Alt case & Normal & Masked \\
\hline HI HF & .90 & .85 & .90 & .95 & .85 & .85 & .35 & .70 \\
\hline HI LF & .90 & .65 & .90 & .85 & .65 & .90 & .35 & .35 \\
\hline LI HF & .75 & .60 & .65 & .80 & .60 & .75 & .0 & .10 \\
\hline LI LF & .55 & .30 & .55 & .45 & .70 & .50 & .10 & .20 \\
\hline High imageability & .90 & .75 & .90 & .90 & .75 & .88 & .35 & .53 \\
\hline Low imageability & .65 & .45 & .60 & .63 & .65 & .63 & .05 & .18 \\
\hline Imageability difference & .25 & .30 & .30 & .28 & .10 & .25 & .30 & .35 \\
\hline High frequency & .83 & .73 & .78 & .88 & .73 & .80 & .18 & .40 \\
\hline Low frequency & .73 & .48 & .73 & .65 & .68 & .70 & .23 & .28 \\
\hline Frequency difference & .10 & .25 & .05 & .23 & .05 & .10 & -.05 & .13 \\
\hline Semantic errors/descriptions & .08 & .03 & .04 & .04 & .0 & .08 & .0 & .38 \\
\hline
\end{tabular}

Alt case: alternating case. Data expressed as a proportion of trials including self-corrections and semantic errors on trials including other error types. 
2.3.2.3. Discussion. The masked condition increased the rate of semantic errors/descriptions for Dysphasic-1, indicating that, under the right circumstances, he showed this cardinal feature of deep dyslexia: indeed, he made more semantic errors than the other patients, in line with his very severe phonological output deficits. Masked presentation reduced Dysphasic-1's attempts to generate phonology from orthography and revealed that he could activate the broad meaning of words he could not read aloud. Dysphasic-1 was actually more accurate at reading masked than unmasked words, suggesting that his attempts to generate phonology from orthography were so inaccurate that they impaired his reading. This finding is consistent with the view that orthography can activate the broad meaning of words directly, independently of phonology. Harm and Seidenberg (2004) recently proposed a computational model of reading in which the meanings of printed words are computed through constraints from two pathways: direct connections from $\mathrm{O} \rightarrow \mathrm{S}$ and indirect connections via phonology $(\mathrm{O} \rightarrow \mathrm{P} \rightarrow \mathrm{S})$. In a patient like Dysphasic-1 with severe phonological impairment, the direct $\mathrm{O} \rightarrow \mathrm{S}$ pathway may be critical for residual reading abilities. Mappings from $\mathrm{O} \rightarrow \mathrm{S}$ are arbitrary: as reading via this route cannot benefit from shared structure between items, this pathway should be highly sensitive to word frequency. In line with this prediction, Dysphasic-1's improvement with masked presentation was almost entirely accounted for by high frequency items. Like patients with "word meaning deafness", Dysphasic1 showed better comprehension of written than spoken words; this pattern has also been taken as evidence of a role for direct $\mathrm{O} \rightarrow \mathrm{S}$ connections in reading (Kohn \& Friedman, 1986; Shelton \& Weinrich, 1997).

\section{General discussion}

The 'primary systems' hypothesis suggests that the various deep syndromes - deep dyslexia, dysphasia and dysgraphia result at least in part from a general phonological impairment, which increases the reliance of each task on word meaning (e.g., Patterson \& Marcel, 1992; Wilshire \& Fisher, 2004). These syndromes should therefore show considerable overlap. This prediction is difficult to evaluate on the basis of previous work because patients with these various deep syndromes have been examined separately in different studies. To address this problem, we directly compared four patients who showed at least one of the deep syndromes. All of the patients were tested using the same repetition, reading and spelling to dictation tasks to establish if some of the characteristics of the deep syndromes (i.e., lexicality and imageability effects) could be observed even in tasks in which semantic errors were not observed. In addition, we utilised a comprehensive battery of neuropsychological tests to examine the status of phonological processing and other 'primary systems' such as semantic, auditory input and visual processing across the four cases. This allowed us to establish whether the patients had a common underlying impairment of phonology and whether differences in other primary systems might account for the appearance of semantic errors in some tasks and not others. Finally, we employed experimental manipulations of repetition and reading that were designed to reduce the extent to which these additional primary systems (auditory input and visual processing, respectively) could constrain phonological output. If all of the deep syndromes share a central phonological deficit, these manipulations should elicit semantic errors in additional tasks: for example, patients with deep dyslexia should show the symptoms of deep dysphasia when the effect of spoken input on repetition is weakened.There were four key findings that provided support for the primary systems hypothesis:

(1) All of the patients had substantial phonological production deficits across different tasks, including repetition, reading aloud and spoken picture naming. They also showed severe deficits in tasks that required the manipulation of phonology, such as phoneme addition and deletion. These findings are consistent with the hypothesis that a common phonological deficit underlies the different deep syndromes.

(2) The patients' phonological deficits impacted on repetition, reading aloud and spelling to dictation in similar ways. Some of the characteristics of the deep syndromes - namely strong effects of lexicality and imageability on accuracy were typically observed in all of the tasks, regardless of whether semantic errors occurred or not. Both the deep dysphasic and the deep dyslexic patients made some semantic errors in spelling to dictation. These findings indicate that the different deep syndromes may partially overlap.

(3) Although there were clear differences between the patients in terms of whether semantic errors were observed in particular tasks, these were explicable in terms of the contribution of additional primary systems-most crucially, auditory input processing. The patients who showed semantic errors in repetition tasks performed poorly at tasks that tapped phonological input processing. In contrast, the deep dyslexics who did not make these errors performed comparatively well in tasks that required spoken input to be analysed. These findings can be explained in terms of the highly systematic relationship between auditory input and phonological output. The good auditory input analysis of the deep dyslexics may have prevented semantic errors in repetition, despite these patients' clear phonological deficits, because the spoken form of the word tightly specified the appropriate phonological output.

(4) Semantic errors were elicited in additional tasks through the use of experimental manipulations that disrupted the contribution of additional primary systems-i.e., auditory processing in repetition and visual processing in reading. The deep dyslexic patients showed the characteristics of deep dysphasia under conditions in which the translation of auditory input to phonological output was difficult (i.e. the repetition of single items following a delay filled with articulatory suppression or the immediate repetition of a pair of items). Both patients showed substantially poorer accuracy and larger imageability effects for delayed/paired repetition compared with standard repetition, and Dyslexic1 also made semantic errors. This supports the view that the deep syndromes share a central phonological deficit and that variation between them can be explained in terms of the con- 
straints provided by additional primary systems. Similarly, a deep dysphasic patient with extremely poor phonological output (Dysphasic-1) made semantic errors in reading aloud when words were presented through a visual mask. This manipulation may have discouraged his attempts to generate phonology from orthography despite his inability to do so accurately (see below).

\subsection{The primary systems hypothesis}

The primary systems hypothesis suggests that tasks such as reading, repetition and spelling to dictation are accomplished through interactions between "primary systems", for example, phonology and semantics, which underpin language processing in general (as well as some non-language tasks). For example, reading aloud is thought to result from interactivity amongst three primary systems: phonology, orthography (or vision) and semantics (Patterson \& Lambon Ralph, 1999; Plaut et al., 1996). Similarly, repetition tasks might be underpinned by interactivity within these same phonological and semantic systems (Patterson, Graham, \& Hodges, 1994; see Martin \& Saffran, 1997, for a similar perspective). Disorders of repetition, reading and spelling to dictation are not thought to result from damage to task-specific routes such as grapheme-phoneme conversion in reading aloud. Instead, deep dysphasia, dyslexia and dysgraphia should overlap to a considerable degree because a severe phonological impairment is thought to be central to all of these syndromes. Although not all deep dyslexic patients make semantic errors in immediate word repetition and not all deep dysphasic patients show semantic errors in standard word reading tasks, differences between these conditions may reflect the contribution of additional primary systems, namely auditory processing in repetition and orthographic/visual input in reading.

This framework can account for the full range of symptoms in deep dyslexia/dysphasia/dysgraphia in a parsimonious way due to the fact that the semantic and phonological systems are highly interactive (Wilshire \& Fisher, 2004; also see Martin \& Saffran, 1992 , for a similar interactive account). When phonological processing is severely impaired, the semantic system plays a greater role in reading, repetition and spelling. Phonologically impaired patients might make semantic errors in these tasks due to their increased reliance on word meaning, together with a reduced influence of phonology which would prevent such responses. Phonologically impaired patients are also highly sensitive to lexicality because they struggle to produce nonwords which derive little support from the semantic system. Similarly, abstract words are difficult for these patients because they have weaker semantic representations than more imageable items: they are associated with a smaller number of semantic features (Plaut \& Shallice, 1993) and/or their meaning depends to a greater extent on sentential context whereas words to read/repeat are typically presented singularly (Schwanenflugel \& Shoben, 1983; Schwanenflugel \& Stowe, 1988). Consequently, phonologically impaired patients show large imageability effects in language tasks. Normal participants also show effects of imageability on reaction times in reading/repetition (Strain, Patterson, \& Seidenberg, 1995; Tyler, Voice, \& Moss, 1996)—however, the healthy system eventu- ally derives the correct phonology of all items, regardless of the degree of semantic support.

\subsection{Explaining task differences}

The findings reviewed above support the "primary systems" view that deep dysphasia, dyslexia and dysgraphia result from severe impairment to a common phonological system, producing overlap between these conditions. Our results also suggest that differences between these syndromes can be explained with reference to other primary systems - in particular, the status of auditory processing is critical in deep dysphasia. Semantic errors in repetition were only observed when auditory input was unable to constrain spoken output either due to poor phonological input processing (for the deep dysphasics) or as a result of task manipulations that inserted irrelevant phonology between the input and output (for Dyslexic-1). This proposal is consistent with the computational model of Plaut and Kello (1999), in which phonological representations result from the interaction of auditory input, articulatory codes and semantic memory. In repetition tasks, both auditory input and semantic memory help to constrain phonological production although auditory input plays a particularly critical role because it has a highly systematic relationship with phonology. This tight coupling between auditory input and phonological processing could explain why aphasic patients typically show a correlation between performance on phonological 'input' and 'output' tasks: the ability to process auditory input will ameliorate the effects of phonological impairment (Martin \& Saffran, 1998, 2002). Within this framework, the symptoms of deep dysphasia might occur after severe damage to phonological representations only for those patients who are unable to use auditory processing to overcome their difficulties (e.g. Dysphasics-1 and 2). Deep dyslexic patients may not show the characteristics of deep dysphasia in the immediate repetition of single items, despite marked phonological impairment that elicits deep dyslexia, because their good auditory processing eliminates the opportunity for semantic errors.

In oral reading, in contrast, we did not observe a strong association between semantic errors and visual/orthographic deficits. Indeed, many deep dyslexics have good lexical decision suggesting that their ability to process orthography is not markedly impaired when phonological production is not required (Coltheart, 1980). In addition, deep and phonological dyslexic patients appear to be relatively insensitive to degradation of the orthographic input; for example, they are unaffected by the use of alternating case and vertical presentation (Saffran \& Marin, 1977). In our study too, reading manipulations had little impact on reading accuracy for Dyslexic-2, Dysphasic-2 and Dysphasic-1 although Dyslexic-1 did perform more poorly in the alternating case condition, possibly because there was a spatialattentional component to his reading disorder (Dysphasic-1's reading errors are considered below).

One explanation of this difference between deep dysphasia and dyslexia centres on the nature of the mapping in repetition and reading (Croot, Patterson, \& Hodges, 1998). There is a highly systematic relationship between the sounds of words and their phonology, which is learned at an early age. Auditory pro- 
cessing is likely to play a central role in the acquisition of phonology, and consequently, auditory impairment may harm phonological processing. In contrast, the mapping between orthography and phonology is less consistent and is acquired later in childhood, after phonological representations have already been established: as a result, orthography plays a less important role in phonological processing. Another difference between reading and repetition concerns the transience of the input: the processing of auditory verbal input may be particularly vulnerable in aphasia because the sound signal constantly changes; in contrast, patients may be able to compensate for mild visual deficits by processing static orthographic stimuli for longer.

This difference between the contribution of auditory processing in repetition and visual/orthographic processing in reading is one of degree. There is a semi-regular relationship between letters and sounds and consequently, orthographic input will help to constrain phonological output in reading tasks to some extent. Severe visual deficits (especially combined with limited presentation time) can produce the symptoms of deep dyslexia (Buxbaum \& Coslett, 1996; Coslett \& Saffran, 1989; Lambon Ralph, Hesketh, \& Sage, 2004). Therefore, both deep dysphasia and dyslexia can be understood in terms of a phonological impairment interacting with semantics and auditory/visual processing.

Perhaps the biggest challenge to the primary systems account of the current data set comes from Dysphasic-1 who did not make semantic errors in standard single word reading despite having the poorest phonological production across tasks. One possible explanation is that semantic errors in Dysphasic-1's oral reading were overridden by his persistent attempts to generate phonology from orthography. Dysphasic-1's spontaneous reading strategy was unusual as he continued to try to sound out letters despite his inability to generate the correct phonology. This strategy may have been a response to his speech and language therapy that had attempted to retrain grapheme-phoneme correspondences. The masked presentation discouraged Dysphasic-1 from using this strategy and greatly increased the rate of semantic errors/descriptions, revealing that, as in deep dyslexia, his oral reading was reliant on word meaning (as predicted by the primary systems hypothesis). Dysphasic-1 was actually more accurate at reading masked than unmasked words, suggesting that his attempts to generate phonology from written words were so inaccurate that they impaired his reading. These findings highlight the need to be cautious when interpreting single case studies which can be greatly influenced by individual differences in the way a task is attempted. Such caution is certainly required when a single feature - such as the presence of semantic errors - is taken as the defining feature of a syndrome.

\section{Conclusion}

This study supports the view that deep dyslexia, dysphasia and dysgraphia share a common phonological deficit. The emergence of semantic errors in reading and repetition is also influenced by the status of other primary systems such as vision and auditory processing which contribute to these tasks. The degree to which these additional systems are critical for the emer- gence of the deep syndromes depends on the systematicity of the mapping between these inputs and phonology. In the repetition task, the tight coupling between auditory input and phonology means that good auditory processing can overcome even severe phonological impairment to eliminate semantic errors.

\section{Acknowledgements}

We would like to thank Kath Mumby for providing some of the background assessments. The work was supported by an IBSC grant from the NIMH (MH64445), an RCUK fellowship awarded to E. Jefferies and a Health Foundation mid-career award to K. Sage (1727.2270).

\section{Appendix A. Error coding}

1. Semantic errors. This category included all of the following except (where otherwise stated): coordinate (lion $\rightarrow$ "zebra"), superordinate (lion $\rightarrow$ "animal"), associative (lion $\rightarrow$ "safari"), descriptive (lion $\rightarrow$ "dangerous animal"), semantic rejected (lion $\rightarrow$ "tiger, no"), semantic then phonological/deviational (lion $\rightarrow$ "teiber"), semantic and phonological (snail $\rightarrow$ "snake"), phonological then semantic (calt $\rightarrow$ "horse" [from colt]) and, for picture naming only, semantic/visual (pretzel $\rightarrow$ "rope").

2. Morphological/derivational errors. Horse $\rightarrow$ "horses"; statement $\rightarrow$ "state".

3. Phonological/visual errors. On tasks requiring a spoken response (e.g., oral picture naming, reading, repetition), some of the target phonemes were preserved. On tasks requiring a written response (written picture naming; spelling to dictation), some of the target letters were produced. The following subtypes were coded: phonologically close words preserving at least half of the target phonemes (lion $\rightarrow$ "line"/LINE); phonologically distant words that contained at least one target phoneme (but fewer than 50\%; lion $\rightarrow$ "late"/LATE); phonologically close nonwords preserving at least half of the target phonemes (lion $\rightarrow$ "lipe"/LIPE); phonologically distant words with at least one target phoneme (lion $\rightarrow$ "laip"/LAPE); for spoken responses only, partial sounding out (lion $\rightarrow$ "l...1..."). As noted elsewhere, the tables show close and distant errors (collapsing across words and nonwords) and lexicality of response (collapsing across close/distant phonological errors and unrelated responses).

4. Unrelated responses. Including words (tiger $\rightarrow$ "straw") and nonwords (tiger $\rightarrow$ "braw").

5. Perseveration of previous response. Both complete (tiger $\rightarrow$ "lion", bus $\rightarrow$ "lion") and partial (tiger $\rightarrow$ "lion", bus $\rightarrow$ "lie").

6. Omission. Including failures to respond and tiger $\rightarrow$ "no" or "don't know".

7. Other.

\section{References}

Basso, A., \& Paulin, M. (2003). Semantic errors in naming, repetition, spelling and drawing from memory: A new Italian case. Neurocase, 9, 109-117. 
Beauvois, M., \& Derouesne, J. (1979). Phonological alexia: Three dissociations. Journal of Neurology Neurosurgery and Psychiatry, 42, 1115-1124.

Beland, R., \& Mimouni, Z. (2001). Deep dyslexia in the two languages of an Arabic/French bilingual patient. Cognition, 82, 77-126.

Binder, J. R., Westbury, C. F., McKiernan, K. A., Possing, E. T., \& Medler, D. A. (2005). Distinct brain systems for processing concrete and abstract concepts. Journal of Cognitive Neuroscience, 17, 905-917.

Bisiacchi, P. S., Cipolotti, L., \& Denes, G. (1989). Impairment in processing meaningless verbal material in several modalities: The relationship between short-term memory and phonological skills. Quarterly Journal of Experimental Psychology, 42, 293-319.

Bozeat, S., Lambon Ralph, M. A., Patterson, K., Garrard, P., \& Hodges, J. R. (2000). Non-verbal semantic impairment in semantic dementia. Neuropsychologia, 38, 1207-1215.

Bub, D., Black, S., Howell, J., \& Kertesz, A. (1987). Speech output processes and reading. In M. Coltheart, G. Sartori, \& Job (Eds.), The cognitive neuropsychology of language. Erlbaum.

Buchanan, L., Hildebrandt, N., \& MacKinnon, G. E. (1994). Phonological processing of non-words by a deep dyslexic patient: A rowse is implicitly a rose. Journal of Neurolinguistics, 8, 163-181.

Buchanan, L., Hildebrandt, N., \& MacKinnon, G. E. (1996). Phonological processing of nonwords in deep dyslexia: Typical and independent? Journal of Neurolinguistics, 9, 113-133.

Buchanan, L., McEwen, S., Westbury, C., \& Libben, G. (2003). Semantics and semantic errors: Implicit access to semantic information from words and nonwords in deep dyslexia. Brain and Language, 84, 65-83.

Buxbaum, L. J., \& Coslett, H. B. (1996). Deep dyslexic phenomena in a letterby-letter reader. Brain and Language, 54, 136-167.

Cappa, S. F., Ielasi, W., Tempini, M. L. G., \& Miozzo, A. (1996). Deep dysphasia: A study of errors in oral and written production. Neurocase, 2, 213219.

Caramazza, A., Miceli, G., \& Villa, G. (1986). The role of the (output) phonological buffer in reading, writing and repetition. Cognitive Neuropsychology, $3,37-76$.

Colangelo, A., Buchanan, L., \& Westbury, C. (2004). Deep dyslexia and semantic errors: A test of the failure of inhibition hypothesis using a semantic blocking paradigm. Brain and Cognition, 54, 232-234.

Colangelo, A., Stephenson, K., Westbury, C., \& Buchanan, L. (2003). Word associations in deep dyslexia. Brain and Cognition, 53, 166-170.

Coltheart, M. (1980). Deep dyslexia: A review of the syndrome. In M. Coltheart, K. Patterson, \& J. C. Marshall (Eds.), Deep dyslexia. London: Routledge and Kegan Paul.

Coslett, H. B., \& Saffran, E. M. (1989). Evidence for preserved reading in pure alexia. Brain, 112, 327-359.

Crisp, J., \& Lambon Ralph, M. A. (2006). Unlocking the nature of the phonological-deep dyslexia continuum: The keys to reading aloud are in phonology and semantics. Journal of Cognitive Neuroscience, 18, 348-362.

Croot, K., Patterson, K., \& Hodges, J. R. (1998). Single word production in nonfluent progressive aphasia. Brain and Language, 61, 226-273.

Dickerson, J., \& Johnson, H. (2004). Sub-types of deep dyslexia: A case study of central deep dyslexia. Neurocase, 10, 39-47.

Farah, M. J., Stowe, R. M., \& Levinson, K. L. (1996). Phonological dyslexia: Loss of a reading-specific component of the cognitive architecture? Cognitive Neuropsychology, 13, 849-868.

Franklin, S., Turner, J. E., \& Ellis, A. W. (1992). The ADA comprehension battery. York, UK: York Human Neuropsychology Laboratory.

Friedman, R., \& Kohn, S. E. (1990). Impaired activation of the phonological lexicon: Effects upon oral reading. Brain and Language, 38, 278297.

Friedman, R. B. (1996). Recovery from deep alexia to phonological alexia: Points on a continuum. Brain and Language, 52, 114-128.

Gold, B. T., \& Kertesz, A. (2001). Phonologically related lexical repetition disorder: A case study. Brain and Language, 77, 241-265.

Goldblum, M. C. (1980). Auditory analogue of deep dyslexia. Experimental Brain Research, Supplement II: Hearing and Speech, 397-405.

Hanley, J. R., \& Kay, J. (1997). An effect of imageability on the production of phonological errors in auditory repetition. Cognitive Neuropsychology, 14 1065-1084.
Harm, M. W., \& Seidenberg, M. S. (2004). Computing the meanings of words in reading: Cooperative division of labor between visual and phonological processes. Psychological Review, 111, 662-720.

Hillis, A., \& Caramazza, A. (1991). Mechanisms for accessing lexical representations for output: Evidence from a category specific semantic deficit. Brain and Language, 40, 106-144.

Howard, D., \& Franklin, S. (1988). Missing the meaning? A cognitive neuropsychological study of the proecssing of words by an aphasic patient. Cambridge, MA: MIT Press.

Howard, D., \& Patterson, K. (1992). Pyramids and palm trees: A test of semantic access from pictures and words. Bury St. Edmunds, Suffolk: Thames Valley Test Company.

Jefferies, E., Crisp, J., \& Lambon Ralph, M. A. (2006). The impact of phonological or semantic impairment on delayed auditory repetition: Evidence from stroke aphasia and semantic dementia. Aphasiology, 20, 963-992.

Jones, G. V. (1985). Deep dyslexia, imageability and ease of prediction. Brain and Language, 24, 1-19.

Katz, R. B., \& Goodglass, H. (1990). Deep dysphasia: Analysis of a rare form of repetition disorder. Brain and Language, 39, 153-185.

Kay, J., Lesser, R., \& Coltheart, M. (1992). Psycholinguistic Assessments of Language Processing in Aphasia (PALPA). Hove, UK: Lawrence Erlbaum Associates.

Kohn, S. E., \& Friedman, R. B. (1986). Word-meaning deafness: A phonologicalsemantic dissociation. Cognitive Neuropsychology, 3, 291-308.

Lambon Ralph, M. A., Hesketh, A., \& Sage, K. (2004). Implicit recognition in pure alexia: The Saffran effect-a tale of two systems or two procedures? Cognitive Neuropsychology, 21, 401-421.

Lezak, M. (1976). Neuopsychological assessment. New York: Oxford University Press.

Majerus, S., Lekeu, F., Van er Linden, M., \& Salmon, E. (2001). Deep dysphasia: Further evidence on the relationship between phonological short-term memory and language processing impairments. Cognitive Neuropsychology, $18,385-410$

Marshall, J. C., \& Newcombe, F. (1973). Patterns of paralexia: A psycholinguistic approach. Journal of Psycholinguistic Research, 2, 175-199.

Martin, N., Dell, G. S., Saffran, E. M., \& Schwartz, M. F. (1994). Origins of paraphasias in deep dysphasia: Testing the consequences of a decay impairment to an interactive spreading activation model of language. Brain and Language, 47, 609-660.

Martin, N., \& Saffran, E. M. (1992). A computational account of deep dysphasia: Evidence from a single case-study. Brain and Language, 43, 240274

Martin, N., \& Saffran, E. M. (1997). Language and auditory-verbal short-term memory impairments: Evidence for common underlying processes. Cognitive Neuropsychology, 14, 641-682.

Martin, N., \& Saffran, E. M. (1998). The relationship between input and output phonology: Evidence from aphasia. Brain and Language, 65, 225-228.

Martin, N., \& Saffran, E. M. (2002). The relationship of input and output phonological processing: An evaluation of models and evidence to support them. Aphasiology, 16, 107-150.

Martin, N., Saffran, E. M., \& Dell, G. S. (1996). Recovery in deep dysphasia: Evidence for a relation between auditory-verbal STM capacity and lexical errors in repetition. Brain and Language, 52, 83-113.

Martin, R. C., Lesch, M. F., \& Bartha, M. C. (1999). Independence of input and output phonology in word processing and short-term memory. Journal of Memory and Language, 41, 3-29.

Michel, F., \& Andreewsky, E. (1983). Deep dysphasia: An analog of deep dyslexia in the auditory modality. Brain and Language, 18, 212-223.

Morton, J., \& Patterson, K. (1980). A new attempt at an interpretation or an attempt at a new interpretation. In M. Coltheart, K. Patterson, \& J. C. Marshall (Eds.), Deep dyslexia (pp. 22-47). London: Routledge and Kegan Paul.

Newton, P. K., \& Barry, C. (1997). Concreteness effects in word production but not word comprehension in deep dyslexia. Cognitive Neuropsychology, 14, 481-509.

Nolan, K. A., \& Caramazza, A. (1982). Modality independent impairments in word processing in a deep dyslexic patient. Brain and Language, 16, 237-264. 
Nolan, K. A., \& Caramazza, A. (1983). An analysis of writing in a case of deep dyslexia. Brain and Language, 20, 305-328.

Patterson, K., Graham, N., \& Hodges, J. R. (1994). The impact of semantic memory loss on phonological representations. Journal of Cognitive Neuroscience, 6, 57-69.

Patterson, K., \& Lambon Ralph, M. A. (1999). Selective disorders of reading? Current Opinion in Neurobiology, 9, 235-239.

Patterson, K., Suzuki, T., \& Wydell, T. N. (1996). Interpreting a case of Japanese phonological alexia: The key is in phonology. Cognitive Neuropsychology, $13,803-822$.

Patterson, K. E., \& Marcel, T. (1977). Aphasia, dyslexia and the phonological coding of written words. Quarterly Journal of Experimental Psychology, 29, 307-318.

Patterson, K. E., \& Marcel, T. (1992). Phonological ALEXIA or PHONOLOGICAL alexia? In J. Alegria, J. Holdender, J. Junca de Morais, \& M. Radean (Eds.), Analytic approaches to human cognition. Amsterdam: Elsevier Science Publishers.

Plaut, D., McClelland, J. L., Seidenberg, M., \& Patterson, K. (1996). Understanding normal and impaired word reading: Computational principles in quasi-regular domains. Psychological Review, 103, 56-115.

Plaut, D. C., \& Kello, C. T. (1999). The emergence of phonology from the interplay of speech comprehension and production: A distributed connectionist approach. In B. MacWhinney (Ed.), The emergence of language. Mahweh, NJ: Erlbaum.

Plaut, D. C., \& Shallice, T. (1993). Deep dyslexia: A case-study of connectionist neuropsychology. Cognitive Neuropsychology, 10, 377-500.

Raven, J. C. (1962). Coloured progressive matrices sets $A, A B, B$. London: $\mathrm{H}$. K. Lewis.

Saffran, E. M. (1980). Reading in deep dyslexia is not ideographic. Neuropsychologia, 18, 219-223.

Saffran, E. M., \& Marin, O. S. M. (1977). Reading without phonology: Evidence from aphasia. Quarterly Journal of Experimental Psychology, 29, 515525.

Sage, K., \& Ellis, A. W. (2004). Lexical influences in graphemic buffer disorder. Cognitive Neuropsychology, 21, 381-400.
Schwanenflugel, P. J., \& Shoben, E. J. (1983). Differential context effects in the comprehension of abstract and concrete verbal materials. Journal of Experimental Psychology: Learning, Memory and Cognition, 9, 82-102.

Schwanenflugel, P. J., \& Stowe, R. W. (1988). The influence of a sentence context on the processing of abstract and concrete words. Bulletin of the Psychonomic Society, 26, 490-1490.

Shallice, T., \& Warrington, E. K. (1980). Single and multiple component central dyslexic syndromes. In M. Coltheart, K. Patterson, \& J. C. Marshall (Eds.), Deep dyslexia (pp. 119-145). London: Routledge and Kegan Paul.

Shelton, J. R., \& Weinrich, M. (1997). Further evidence of a dissociation between output phonological and orthographic lexicons: A case study. Cognitive Neuropsychology, 14, 105-129.

Strain, E., Patterson, K., \& Seidenberg, M. S. (1995). Semantic effects in single word naming. Journal of Experimental Psychology: Learning, Memory and Cognition, 21, 1140-1154.

Trojano, L., Stanzione, M., \& Grossi, D. (1992). Short-term memory and verbal learning with auditory phonological coding defect: A neuropsychological case study. Brain and Cognition, 18, 12-33.

Tyler, L. K., Voice, J. K., \& Moss, H. E. (1996). The interaction of semantic and phonological processing. In Proceedings of the 18th Annual Conference of the Cognitive Science Society.

Valdois, S., Carbonnel, S., David, D., Rousset, S., \& Pellat, J. (1995). Confrontation of PDP models and dual-route models through the analysis of a case of deep dysphasia. Cognitive Neuropsychology, 12, 681-724.

Warrington, E. K., \& James, M. (1991). The visual object and space perception battery. Bury St. Edmunds, Suffolk: Thames Valley Test Company.

Warrington, E. K., McKenna, P., \& Orpwood, L. (1998). Single word comprehension: A concrete and abstract word synonym test. Neuropsychological Rehabilitation, 8, 143-154.

Westbury, C., \& Buchanan, L. (2006). Toward a frontal lobe disconnection model of deep dyslexia: The role of semantic feedback in phonological false memories. Journal of Neurolinguistics, 19, 124-156.

Wilshire, C. E., \& Fisher, C. A. (2004). Phonological" dysphasia: A crossmodal phonological impairment affecting repetition, comprehension, and production. Cognitive Neuropsychology, 21, 187-210. 